\title{
The Application of Mandibular Nerve Blocks by Dental Specialists in Bulgaria - Anonymous Survey
}

\author{
Rusi Argirov*, Petia Pechalova \\ Department of oral surgery, Faculty of dental medicine, Medical University of Plovdiv, Bulgaria
}

*Corresponding Author: Rusi Argirov, Department of oral surgery, Faculty of dental medicine, Medical University of Plovdiv, Bulgaria, Email: russsi144@gmail.com

\begin{abstract}
Mandibular nerve blocks are crucial in providing anesthesia in the posterior segments of the mandible. Many different methods are described in the literature and it's important for the dental practitioner to be familiar with at least a couple of them so that he can choose between them depending on the clinical case.

The purpose of this survey is to acquire data about the usage of mandibular nerve block in Bulgaria. In order to acquire the data an anonymous questionnaire was filled by 1008 dental practitioners in the country, 100 of which stated that they had a dental specialty

The results of the survey show, that $29 \%$ of the dental specialists use mandibular nerve blocks in their daily practice, $18 \%$ don't and $53 \%$ use it in some of their cases. The participants in the survey stated that they prefer the Weisbrem technique (42\%) to the Halsted method (38\%) and only 1 percent stated that they used the Vazirani-Akinosi technique. The rest of the participants (18\%) stated that they didn't implement mandibular nerve blocks in their daily practice. The results show that many of the dental specialists in the country should improve their theoretical and practical skills regarding mandibular nerve blocks so that the patients can receive better pain control and a dental treatment of higher standard.
\end{abstract}

Keywords: mandibular anesthesia, nerve block, dental anesthesia

\section{INTRODUCTION}

The Halsted approach to the inferior alveolar nerve block is the most common method of mandibular block anesthesia to be employed in dental practices around the world. However, because of its inconsistent success rate, other methods are also researched and developed, for example the nerve block employed for the first time by George A.E. Gow-Gates, who in 1973 describes the approach [1]. Such a technique however requires a maximal mouth opening, which is some urgent conditions is not possible for the patient. For that reason in 1960 and in 1977 accordingly, S. Vazirani and J.O. Akinosi [2] describe independently from one another, the closed mouth nerve block. The Weisbrem technique is the mandibular nerve block, which is commonly employed in Bulgaria, because it is extensively thought in the medical universities in the country.

\section{Purpose}

The purpose of this study is to evaluate the usage of the mandibular nerve blocks in Bulgaria by dental practitioners with an acquired dental specialty and more precisely the methods that the dentists frequent as well as the clinical situations, they use them in.

\section{MethodS}

An anonymous questionnaire, containing 15 questions was distributed in Bulgaria. The survey was made through Google Forms and links were sent to dentists all around the country. Paper variants of the questionnaire were also distributed to different practitioners. Till the end of the determined date, 1008 questionnaires were returned. Of all the participants -100 have indicated that they have an acquired dental specialty. The results were processed and analyzed with IMB SPSS 23 and Microsoft Excel.

\section{Results}

From all the participants $(\mathrm{n}=1008)$, only 100 of the dentists have an acquired specialty (9.9\%). Almost all of the specialists are dentist with a working experience of more than 10 years 94\% ( $\mathrm{n}=94)$. From all the dental practitioners that participated in the study $-9(0.9 \%)$ 
indicated that they are now in the middle of specialization. Most of the dental specialists listed that their specialty is Prosthetic dental medicine $-41(4.1 \%)$, followed by the ones that specialize in conservative dentistry and endodontics - $23(2.3 \%)$ and the orthodontists $20(2 \%)$. In fourth place are the oral surgeons -
$16(1.6 \%)$, followed by the ones with general dental medicine $-4(0.4 \%)$ and the smallest group were the specialists in periodontics -3 dentists $(0.3 \%)$. Two of the participants indicated that they have 2 specialties. This analysis includes and the ones that are still in the middle of their qualification. (Figure 1)

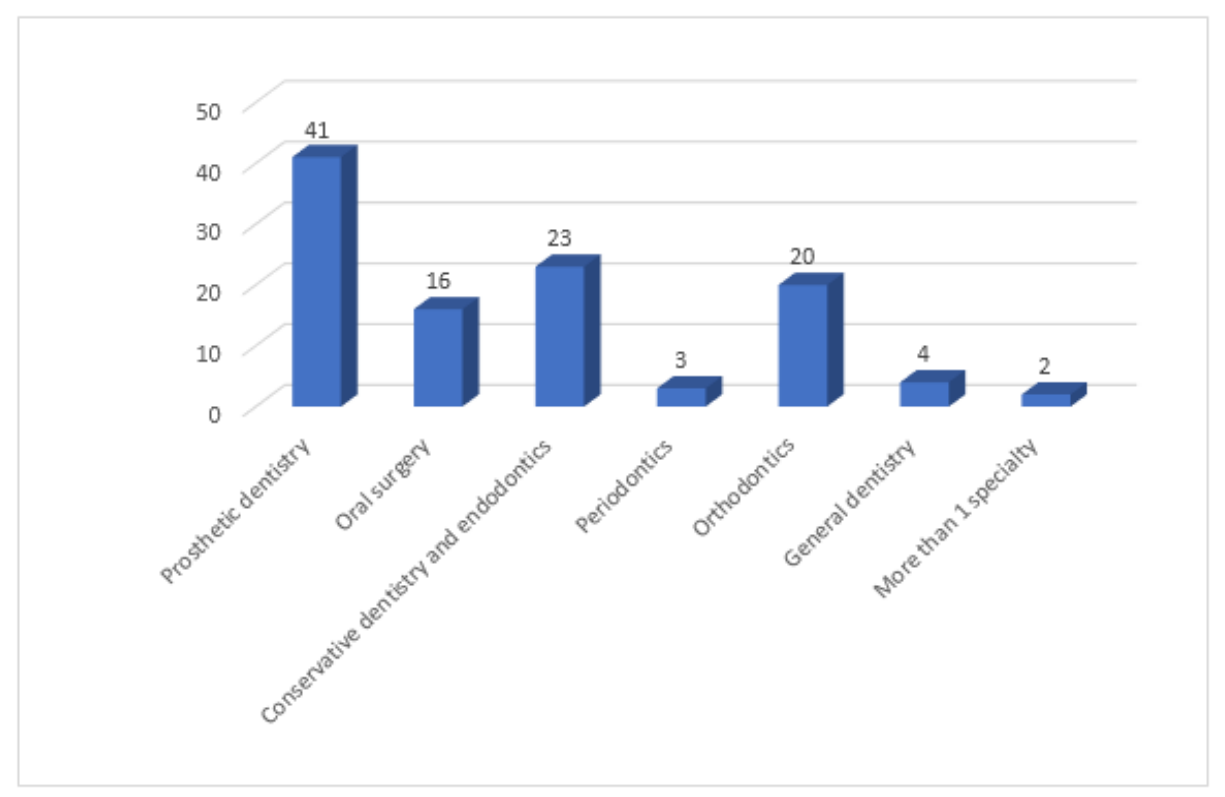

Figure1: The different dental specialties that the participants in the study have

The processing of the data, regarding the workplace of dental specialists, showed that $60 \%(n=60)$ of them work in their own dental practice. Next is the group that work in practice with two practitioners but working independently from one another $-28 \%(\mathrm{n}=28)$.
The specialists that work in a group practice with more than two practitioners are only 3 $(3 \%)$. There are $8(8 \%)$, who work in a Dental Faculty and only one of the practitioners has indicated that he works in a Hospital/Maxillofacial department. The data is showed in Figure 2.

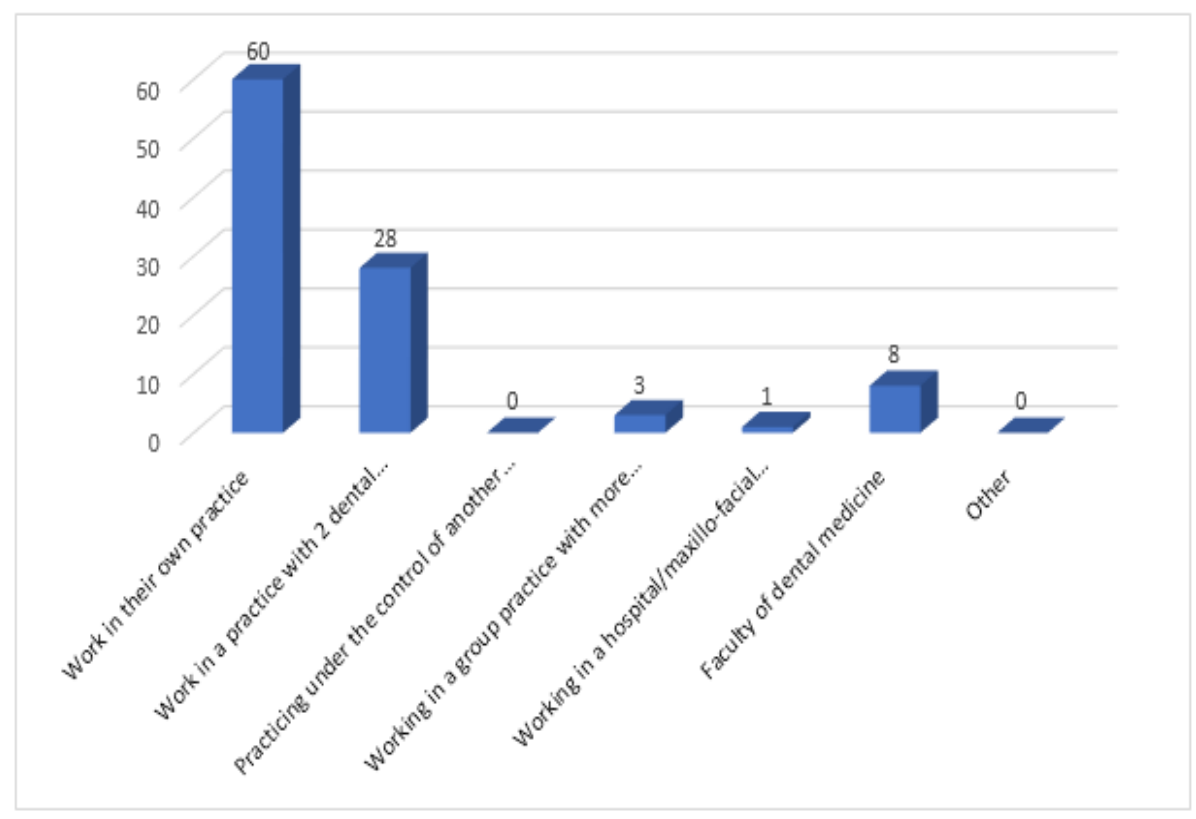

Figure2: Workplace of dental specialists

From all the participants in the survey, 29\% $(n=29)$ use mandibular nerve blocks in their daily practice, $18 \% \quad(\mathrm{n}=18)$ don't and $53 \%$ $(n=53)$ employ block anesthesia of the mandible in some cases. (Figure 3) 


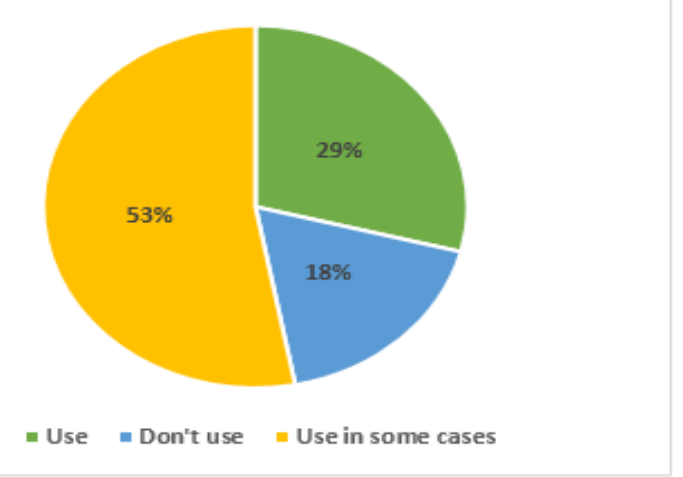

Figure3: The number of clinicians with a specialty that use mandibular nerve blocks in their daily practice.

The surveyed dentists indicate that most of them frequent the Weisbrem (Torusal) mandibular block $-42 \%(n=42)$, followed by the group that usually employ the Halsted classical approach $38 \%(\mathrm{n}=38)$. The Akinosi nerve block technique was employed only by $1 \% \quad(n=1)$ of the participants in the study, while no one marked the Gow-Gates technique. One of the dentists pointed that he had no idea what was the name of the technique, that he used and the rest - 18\% $(n=18)$ indicated that they don't employ mandibular nerve blocks in their practice. (Figure 4)

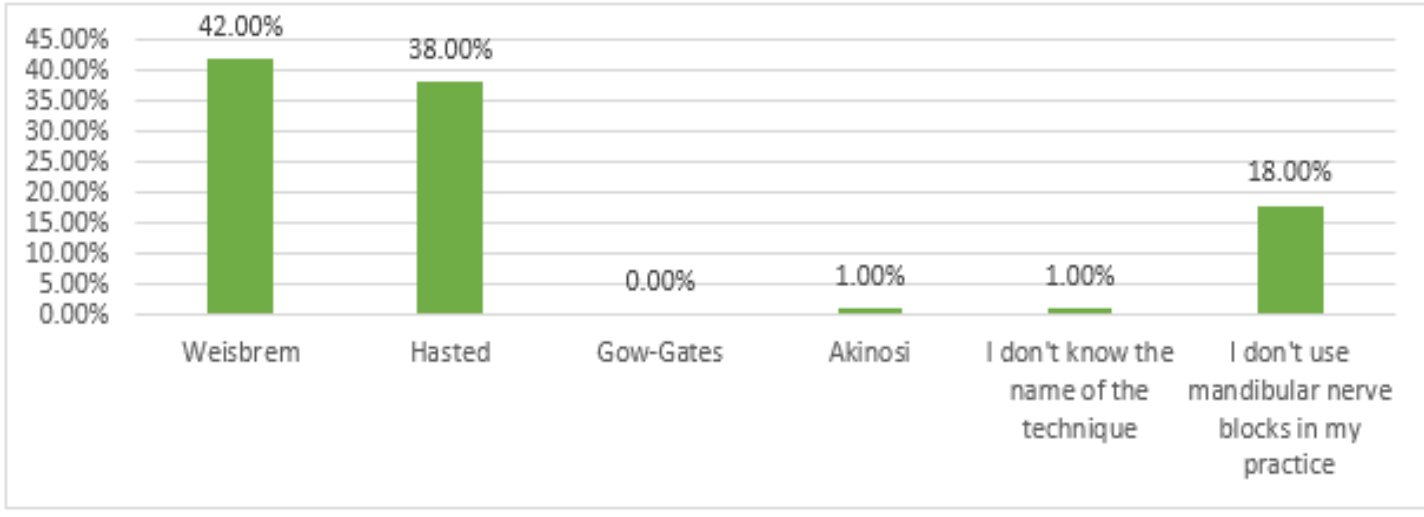

Figure4: Techniques that the clinicians with an acquired specialty most frequently employ in their practice.

There was a question in the survey, about how the clinicians asses the success rate of the mandibular blocks they use. Most of them - 63\% $(n=63)$ marked their blocks as providing quick onset and profound anesthesia during the procedure. Only $12 \%(\mathrm{n}=12)$ have indicated that their blocks don't provide enough anesthesia and the onset is slow, so that a supplementary technique is necessary to fully anesthetize the patient. Seven clinicians $(7 \%)$ listed their mandibular nerve blocks as unsuccessful and needed to substitute the technique with another method. The rest of the dental specialists have marked, that they don't employ mandibular nerve blocks. (Figure 5)

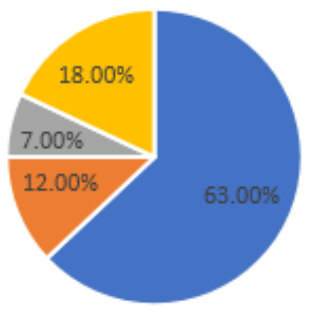

\footnotetext{
- Fast onset with a profound enough anesthetic effect

- Slow onset and insufficient pain control, need for a supplementary technique

- Unsuccessful nerve block, which has to be substituted with another method

= I don't use mandibular nerve blocks
}

Figure5: Assessment of the success rate of the mandibular nerve blocks that dentists with an acquired specialty, use 


\section{DISCUSSION}

In our survey, most of the dental clinicians with an acquired specialty have a working experience of more than 10 years $-94 \%(n=94)$, while a study from 2017 by the American college ${ }^{3}$ for dental prosthodontists shows that from 325 dentists, which specialize in prosthetic dentistry and have their own practice, $47.69 \% \quad(n=155)$ have experience of less than 10 years, $20.31 \%$ $(n=66)$ have a working experience between 10 and 19 years, $16 \%$ have been working between 20 and 29 years in the field, $12 \%$ - between 30 and 39 years and only $4 \%$ have a working experience of more than 40 years.

The most popular technique to anesthetize the inferior alveolar nerve in Bulgaria is the Weisbrem technique, also known as Torusal mandibular nerve block. This technique is rarely employed in western Europe and North America. The second most common technique to anesthetize the mandible is the Hasted technique, as almost no one of the other participants has marked the Gow-Gates or the Akinosi-Vazirani technique. Most authors give similar success rates for the alternatives (GowGates and Akinosi methods) to the classical Halsted approach and also list some advantages to using them. In a study by Diandian Li [4], it is reported that all patients in the groups in which the Gow-Gates $(n=140)$ and the Akinosi technique $(n=140)$ were employed were successfully anesthetized, while in the group of patients, who were anesthetized with the Hasted technique there were 4 cases, in which the technique failed to provide pain control. Jiacai $\mathrm{He}$ [5] carried out a study on mandibular nerve blocks and found out that 38 out of 40 patients, anesthetized with the Akinosi technique, experienced sufficient pain control, while 37 out of 40 in the Halsted group, experienced adequate pain control. The research that Jizhong Lv [6] carried out over 120 people also showed similar results. The patients were divided into two groups- each group comprised of 60 people. All the patients in the first group were anesthetized with the Akinosi-Vazirani technique and the success rate was $100 \%$ while in the second group, the classic Halsted approach was used and 58 of 60 patients were reported as successfully anesthetized. Fei Wang [7] reports 27 out of 30 people as successfully anesthetized with the Gow-Gates and 29 out of 30 in the Halsted group. Jieping Yang [8] had the opposite results as his study showed higher success rate for the Gow-Gates technique (31/32 patients) over the Halsted method (29/32 patients). Martinez [9] and coll. reported a successful Akinosi mandibular nerve block in 23 out of 28 patients while the Halsted method had a higher success rate in their hands -25 out of 28 patients. In all these studies, the Dobbs scale was used for measuring the success rate of the mandibular nerve blocks. In this scale the patients can report 3 stages - stage 1 -slight pain; stage 2 - moderate pain but without the need for supplemental injection and stage 3 Strong pain, requiring additional pain control. For a mandibular nerve block to be considered successful, the patients should evaluate the pain as stage 1 or stage 2 in the Dobbs scale. Fanyuan $\mathrm{Yu}$ and coll. [10] established when comparing the 3 techniques that Gow-Gates has a higher success rate than the classical approach of Hasted $(\mathrm{RR}=1.04,95 \% \mathrm{CI}=1.01-1.08, \mathrm{P}=$ 0.02.), however, there was no statistical significance that proved a higher success rate for the Akinosi technique, compared to the Hasted mandibular nerve block.

Eighteen percent of all the participants in our study, indicated that they don't use mandibular nerve blocks in their daily practice. Dental practitioners that avoid using mandibular nerve blocks often use terminal infiltration or periodontal injection in the posterior mandibular region. A study by Rozh M. Hussein [11] is comparing the effect of mandibular nerve blocks and terminal infiltration, when extracting nonvital premolars and molars of the mandible. The results showed that when using buccal and lingual infiltration in $68.2 \%$ of the cases there was an adequate enough pain control for extraction of the teeth. In the other group the anesthesia was done with a Halsted technique and a long buccal nerve block and that was enough to cause sufficient pain control for extraction in all patients (100\%). The anesthetic agent that was used was lidocaine. On the other hand, when handling vital teeth, things are different. The diffusion of articaine through the cortical plate of the bone is much better than that of the lidocaine. Muhammad Zain [12] compared the effect of buccal terminal infiltration in the posterior mandibular region with the effect of mandibular nerve blocks, when the anesthetic agent of choice was articaine. The study included patients with symptomatic first molar of the mandible and in $71.11 \%$ of the cases with buccal infiltration, there was profound enough anesthesia. Only in $64.4 \%$ of the cases that were anesthetized with the Halsted technique there was a successful pain control. So the authors claim that in many 
of the cases, terminal infiltration with articaine can provide sufficient pain control even in the posterior region of the mandible.

\section{CONCLuSion}

A large part of the dental specialists in Bulgaria use only the Hasted and the Weisbrem techniques, when employing mandibular nerve blocks, which demonstrates lack of practical and theoretical knowledge about the alternative techniques. Also, some of the specialists avoid using mandibular nerve blocks because of many factors like low success rate, fear of complications occurring and prefer using terminal infiltration or periodontal injection.

\section{REFERENCES}

[1] Stanley F. Malamed : Handbook of Local Anaesthesia, chapter 14, Techniques of mandibular anaesthesia, Elsevier publishers, N. Delhi, 2007, page 237

[2] Allen L. Sisk: Evaluation of the Akinosi Mandibular Block Technique in Oral Surgery. J Oral Max Fac Surg 1986; Vol. 44, No. 2: 11315.

[3] Kent D. Nash, PhD, KD Nash Enterprises. American College of Prostodontists. Survey of Prostodontists 2017, results of the survey.

[4] Li, D., Nan, X. \& Xie, G. Clinical application and evaluation of three types of blockanesthesia of inferior alveolar nerve. ChineseJournal of Practical Stomatology 2, 36-38 (2009)

[5] He, J. \& Jiang, C. Evaluation of two different methods of inferior alveolar nerve block anesthesia. Acta Universitatis Medicinalis Anhui 35, 281-282 (2000).

[6] Lv, J. \& Zhang, C. Clinical application and evaluation of two types of block anesthesia. Journal OfHeze MedicalCollege 21, 3-5(2009).

[7] Wang, F. \& Feng, C. Applying Gow-Gates mandibular nerve conduction anesthesia to extraction of mandible impacted tooth.J Modern Stomatol 16, 69-70 (2002).

[8] Yang, J., Liu, W. \& Gao, Q. The anesthetic effects of Gow-Gates technique of inferior alveolar nerve block in impacted mandibular third molar extraction. West China Journal of Stomatology 31, 381-384 (2013).

[9] Martínez-González, J. M., Benito-Peña, B., Fernández-Cáliz, F., SanHipólito-Marín, L. \& Peñarrocha-Diago, M. A comparative study of direct mandibular nerve block and the Akinosi technique. Med Oral 8, 143-149 (2003).

[10] Fanyuan, Y. et al. Evaluation of Three Block Anesthesia Methods for Pain Management During Mandibular Third Molar Extraction: A Meta-analysis. Sci. Rep. 7, 40987; doi: 10.103 8/srep40987 (2017).

[11] Rozh M .Hussein, DilmanN.Muhammad, Othman A. Omar. Comparison between infiltration and inferior alveolar nerve block anesthesia in extraction of non-vital mandibular posterior teeth (prospective clinical study). Zanco J. Med. Sci., Vol. 18, No. (3), 2014

[12] Zain M, Khattak SR, Shah SA, Sikandar H, Khattak Y. Comparison of effectiveness of $4 \%$ articaine buccal infiltration versus inferior alveolar nerve block in symptomatic mandibular 1st molar tooth. J Postgrad Med Inst 2015; 29(3): 176-80.

Citation: Rusi Argirov, Petia Pechalova, The Application of Mandibular Nerve Blocks by Dental Specialists in Bulgaria - Anonymous Survey. ARCJournal of Dental science. 2019; 4(4):18-22. doi:dx.doi.org/10.20431/ 2456-0030.0404005.

Copyright: () 2019 Authors. This is an open-access article distributed under the terms of the Creative Commons Attribution License, which permits unrestricted use, distribution, and reproduction in any medium, provided the original author and source are credited. 